\title{
Risk of incident diabetes among patients treated with statins: population based study
}

This Research paper contains an error in the results section of the abstract (BMJ 2013;346:f2610, doi:10.1136/bmj.f2610).

The fourth sentence should have stated that "There was a slightly higher (not "lower") absolute risk with simvastatin (26 outcomes per 1000 person years) compared with pravastatin (23 outcomes per 1000 person years)."

Cite this as: BMJ 2013;347:f4356

๑ BMJ Publishing Group Ltd 2013 